\title{
FINITE GROUPS ACTING ON SEVERI-BRAUER SURFACES
}

\author{
CONSTANTIN SHRAMOV
}

\begin{abstract}
We classify finite groups that can act by automorphisms and birational automorphisms on non-trivial Severi-Brauer surfaces over fields of characteristic zero.
\end{abstract}

\section{Contents}

1. Introduction

2. Semidirect products

3. Central simple algebras

4. Construction of subgroups

5. Automorphisms of prime order

6. Subgroups

7. Field of rational numbers

References

\section{INTRODUCTION}

A Severi-Brauer surface over a field $\mathbb{K}$ is a surface that becomes isomorphic to $\mathbb{P}^{2}$ over the algebraic closure of $\mathbb{K}$. Concerning birational automorphisms of non-trivial SeveriBrauer surfaces (i.e. those that are not isomorphic to $\mathbb{P}^{2}$ over the base field) the following is known.

Theorem 1.1 ([Sh20a, Theorem 1.2(ii)]). Let $S$ be a non-trivial Severi-Brauer surface over a field $\mathbb{K}$ of characteristic zero. Then every finite group acting by birational automorphisms of $S$ is either abelian, or contains a normal abelian subgroup of index 3 .

The following result was proved in [Sh20b]. We denote by $\boldsymbol{\mu}_{n}$ the cyclic group of order $n$.

Theorem 1.2. Let $p \equiv 1(\bmod 3)$ be a prime number. Consider the non-trivial homomorphism $\boldsymbol{\mu}_{3} \rightarrow \operatorname{Aut}\left(\boldsymbol{\mu}_{p}\right)$, and let $G_{p} \cong \boldsymbol{\mu}_{p} \rtimes \boldsymbol{\mu}_{3}$ be the corresponding semidirect product. Then there exists a number field $\mathbb{K}$ and a non-trivial Severi-Brauer surface $S$ over $\mathbb{K}$ such that the group $\operatorname{Aut}(S)$ contains the group $G_{p}$.

Due to the work of I. Dolgachev and V. Iskovskikh [DI09a], a classification of finite groups acting by birational selfmaps of $\mathbb{P}^{2}$ over an algebraically closed field of characteristic zero is available. The purpose of this paper is to give a classification of finite groups acting by birational selfmaps on non-trivial Severi-Brauer surfaces over fields of characteristic zero. We will show that possible finite subgroups of automorphism groups of non-trivial Severi-Brauer surfaces are cyclic groups of certain orders, and semidirect product of cyclic groups with $\boldsymbol{\mu}_{3}$ of certain kind; possible finite subgroups of birational automorphism groups of non-trivial Severi-Brauer surfaces are finite subgroups of automorphism groups together with the group $\boldsymbol{\mu}_{3}^{3}$. 
To formulate a precise assertion, we need to introduce additional notation. Given a positive integer $n$, denote by $\boldsymbol{\mu}_{n}^{*}$ the multiplicative group of residues modulo $n$ that are coprime to $n$. Let $G$ be a semidirect product of $\boldsymbol{\mu}_{n}$ and $\boldsymbol{\mu}_{3}$ corresponding to a homomorphism

$$
\chi: \boldsymbol{\mu}_{3} \rightarrow \boldsymbol{\mu}_{n}^{*} \cong \operatorname{Aut}\left(\boldsymbol{\mu}_{n}\right) .
$$

If $n=\prod p_{i}^{r_{i}}$, where $p_{i}$ are pairwise different primes congruent to 1 modulo 3 , we say that $G$ is balanced if the compositions of $\chi$ with all natural projections $\boldsymbol{\mu}_{n}^{*} \rightarrow \boldsymbol{\mu}_{p_{i}}^{*}$ are injective. An example of such a semidirect product is the group $\boldsymbol{\mu}_{3} \cong \boldsymbol{\mu}_{1} \rtimes \boldsymbol{\mu}_{3}$ itself. We point out that a balanced semidirect product of a given order may be not unique. The reader is referred to \$2 for details.

Theorem 1.3. Let $n$ be a positive integer, and let $G$ be a finite group. The following assertions hold.

(i) Let $n$ be a positive integer. There exists a field $\mathbb{K}$ of characteristic zero and a non-trivial Severi-Brauer surface over $\mathbb{K}$ such that the group $\operatorname{Bir}(S)$ of birational automorphisms of $S$ contains an element of order $n$ if and only if $n=3^{r} \prod p_{i}^{r_{i}}$, where $p_{i}$ are primes congruent to 1 modulo 3 , and $r \leqslant 1$ (in other words, $n$ is not divisible by 9 and not divisible by primes congruent to 2 modulo 3$)$. In this case the group $\operatorname{Aut}(S)$ contains an element of order $n$ as well.

(ii) Let $G$ be a finite group. There exists a field $\mathbb{K}$ of characteristic zero and a nontrivial Severi-Brauer surface over $\mathbb{K}$ such that the group Aut $(S)$ contains a subgroup isomorphic to $G$ if and only if there is a positive integer $n$ divisible only by primes congruent to 1 modulo 3 such that $G$ is isomorphic either to $\boldsymbol{\mu}_{n}$, or to $\boldsymbol{\mu}_{3 n}$, or to a balanced semidirect product $\boldsymbol{\mu}_{n} \rtimes \boldsymbol{\mu}_{3}$, or to the direct product $\boldsymbol{\mu}_{3} \times\left(\boldsymbol{\mu}_{n} \rtimes \boldsymbol{\mu}_{3}\right)$, where the semidirect product is balanced.

(iii) Let $G$ be a finite group. There exists a field $\mathbb{K}$ of characteristic zero and a nontrivial Severi-Brauer surface over $\mathbb{K}$ such that the group $\operatorname{Bir}(S)$ contains a subgroup isomorphic to $G$ if and only if either $G$ is one of the groups listed in assertion (ii), or $G \cong \boldsymbol{\mu}_{3}^{3}$.

One can reformulate Theorem 1.3 as a description of the sets of all finite orders of elements and all finite subgroups of the groups $\operatorname{Aut}(S)$ and $\operatorname{Bir}(S)$, where $S$ varies in the set $\mathcal{S}$ of all non-trivial Severi-Brauer surfaces over all fields of characteristic zero. Namely, denote

$$
\begin{aligned}
\mathcal{A O}= & \left\{n \mid n \in \mathbb{Z}_{\geqslant 1}, \text { and there exists } S \in \mathcal{S}\right. \\
& \text { such that } \operatorname{Aut}(S) \text { contains an element of order } n\}, \\
\mathcal{B O}= & \left\{n \mid n \in \mathbb{Z}_{\geqslant 1}, \text { and there exists } S \in \mathcal{S}\right. \\
& \text { such that } \operatorname{Bir}(S) \text { contains an element of order } n\}, \\
\mathcal{A G}= & \{G|| G \mid<\infty, \text { and there exists } S \in \mathcal{S} \\
& \text { such that } \operatorname{Aut}(S) \text { contains a subgroup isomorphic to } G\}, \\
\mathcal{B G}= & \{G|| G \mid<\infty, \text { and there exists } S \in \mathcal{S} \\
& \text { such that } \operatorname{Bir}(S) \text { contains a subgroup isomorphic to } G\} .
\end{aligned}
$$

Also, denote by $\mathcal{N}$ the set of all positive integers of the form $n=\prod p_{i}^{r_{i}}$, where $p_{i}$ are primes congruent to 1 modulo 3 , and denote

$$
\mathcal{B}=\left\{G \mid G \cong \boldsymbol{\mu}_{n} \rtimes \boldsymbol{\mu}_{3} \text { is a balanced semidirect product for some } n \in \mathcal{N}\right\} \text {. }
$$


In these notation, Theorem 1.3 claims that

$$
\begin{aligned}
& \mathcal{A O}=\mathcal{B O}=\mathcal{N} \cup\{3 n \mid n \in \mathcal{N}\} \\
& \mathcal{A G}=\left\{\boldsymbol{\mu}_{n} \mid n \in \mathcal{N}\right\} \cup\left\{\boldsymbol{\mu}_{3 n} \mid n \in \mathcal{N}\right\} \cup \mathcal{B} \cup\left\{\boldsymbol{\mu}_{3} \times G \mid G \in \mathcal{B}\right\} \\
& \mathcal{B G}=\mathcal{A G} \cup\left\{\boldsymbol{\mu}_{3}^{3}\right\}
\end{aligned}
$$

As we will see in Remark 4.2, every Severi-Brauer surface over a field of characteristic zero has an automorphism of order 3. However, over some fields birational automorphism groups of non-trivial Severi-Brauer surfaces may be rather poor in finite subgroups. For instance, in the case of the field $\mathbb{Q}$ of rational numbers, Theorem 1.3 implies the following simpler classification.

Corollary 1.4. Let $S$ be a non-trivial Severi-Brauer surface over $\mathbb{Q}$, and let $G$ be a finite subgroup of $\operatorname{Bir}(S)$. Then $G$ is a subgroup of $\boldsymbol{\mu}_{3}^{3}$. In particular, $G$ is abelian.

Using the terminology of [Pop14, Definition 1], we see from Corollary 1.4 that the Jordan constant of the birational automorphism group of every non-trivial Severi-Brauer surface over $\mathbb{Q}$ equals 1 , while for a non-trivial Severi-Brauer surface over an arbitrary field of characteristic zero it does not exceed 3 by Theorem 1.1. Note that the Jordan constant of the birational automorphism group of the projective plane over $\mathbb{Q}$ equals 120 by [Yas17, Theorem 1.11]. I do not know if the groups $\boldsymbol{\mu}_{3}^{2}$ or $\boldsymbol{\mu}_{3}^{3}$ can actually be embedded into birational automorphism groups of some non-trivial Severi-Brauer surfaces over $\mathbb{Q}$.

It would be interesting to obtain a complete classification of finite groups acting on each non-trivial Severi-Brauer surface depending on the arithmetic properties of the base field and the correspondning central simple algebra, similarly to what was done for conics in GA13, cf. Bea10. Also, it would be interesting to find out if there exists an (infinite) extension $\mathbb{K}^{\prime}$ of $\mathbb{Q}$ and a non-trivial Severi-Brauer surface $S^{\prime}$ over $\mathbb{K}^{\prime}$ such that the group $\operatorname{Aut}\left(S^{\prime}\right)$ contains all the groups listed in Theorem 1.3)(ii), and $\operatorname{Bir}\left(S^{\prime}\right)$ contains all the groups listed in Theorem 1.3 (iii).

The plan of the paper is as follows. In $\$ 2$ we collect some auxiliary assertions concerning semidirect products of cyclic groups. In $\$ 3$ we gather auxiliary facts concerning SeveriBrauer surfaces and Galois theory. In $\$ 4$ we construct examples of finite groups acting on non-trivial Severi-Brauer surfaces. In \$5 we describe the possible finite orders of automorphisms of non-trivial Severi-Brauer surfaces. In 6 we classify finite subgroups of automorphism groups of non-trivial Severi-Brauer surfaces and prove Theorem 1.3. In \$7 we prove Corollary 1.4.

Notation. Given a field $\mathbb{K}$, we denote by $\overline{\mathbb{K}}$ its algebraic closure. For a variety $X$ defined over $\mathbb{K}$, we denote by $X_{\overline{\mathbb{K}}}$ its extension of scalars to $\overline{\mathbb{K}}$.

Acknowledgements. I am grateful to S. Gorchinskiy, L. Rybnikov, A. Trepalin, and V. Vologodsky for useful discussions. I am also grateful to the referee for helpful comments, and especially for Remark 7.2. Special thanks go to D. Osipov who spotted a gap in a preliminary version of the paper and suggested several improvements of the exposition. I was partially supported by the HSE University Basic Research Program, Russian Academic Excellence Project "5-100", and by the Foundation for the Advancement of Theoretical Physics and Mathematics "BASIS". 


\section{SEMIDIRECT PRODUCTS}

In this section we collect some elementary assertions concerning semidirect products of certain finite cyclic groups.

Let $n$ be a positive integer. Write

$$
n=\prod_{i=1}^{k} p_{i}^{r_{i}},
$$

where $p_{i}$ are pairwise different prime numbers. There is a canonical isomorphism

$$
\boldsymbol{\mu}_{n} \cong \prod_{i=1}^{k} \boldsymbol{\mu}_{n}\left(p_{i}\right)
$$

where $\boldsymbol{\mu}_{n}\left(p_{i}\right) \cong \boldsymbol{\mu}_{p_{i}^{r_{i}}}$ is the $p_{i}$-Sylow subgroup of $\boldsymbol{\mu}_{n}$. For the multiplicative group $\boldsymbol{\mu}_{n}^{*}$ of residues modulo $n$ that are coprime to $n$, one has a canonical isomorphism

$$
\boldsymbol{\mu}_{n}^{*} \cong \prod_{i=1}^{k} \boldsymbol{\mu}_{n}\left(p_{i}\right)^{*},
$$

where $\boldsymbol{\mu}_{n}\left(p_{i}\right)^{*} \cong \boldsymbol{\mu}_{p_{i}^{r_{i}}}^{*}$. If $p_{i}>2$, we have

$$
\boldsymbol{\mu}_{n}\left(p_{i}\right)^{*} \cong \boldsymbol{\mu}_{p_{i}^{r_{i}-1}\left(p_{i}-1\right)},
$$

see for instance [Lan65, § II.2].

Recall that the group $\operatorname{Aut}\left(\boldsymbol{\mu}_{n}\right)$ is canonically identified with $\boldsymbol{\mu}_{n}^{*}$. Thus, for every homomorphism $\chi: \boldsymbol{\mu}_{3} \rightarrow \boldsymbol{\mu}_{n}^{*}$ we can construct a semidirect product $G \cong \boldsymbol{\mu}_{n} \rtimes \boldsymbol{\mu}_{3}$. Vice versa, every semidirect product corresponds to some homomorphism $\chi$.

Definition 2.1. Suppose that $n$ is divisible only by primes congruent to 1 modulo 3, and let $\chi: \boldsymbol{\mu}_{3} \rightarrow \boldsymbol{\mu}_{n}^{*}$ be a homomorphism. We say that $\chi$ is balanced if its composition with each of the projections $\boldsymbol{\mu}_{n}^{*} \rightarrow \boldsymbol{\mu}_{n}\left(p_{i}\right)^{*}$ is an embedding. We say that a semidirect product $G$ of $\boldsymbol{\mu}_{n}$ and $\boldsymbol{\mu}_{3}$ corresponding to the homomorphism $\chi$ is balanced if $\chi$ is balanced.

Example 2.2. Let $n=1$. Then the set of prime divisors of $n$ is empty, and thus Definition 2.1 does not impose any condition on a semidirect product. Thus, the semidirect product $\boldsymbol{\mu}_{3} \cong \boldsymbol{\mu}_{1} \rtimes \boldsymbol{\mu}_{3}$ is balanced.

Example 2.3. Let $p$ be a prime number congruent to 1 modulo 3 . Then the group $G_{p}$ described in Theorem 1.2 is a balanced semidirect product of $\boldsymbol{\mu}_{p}$ and $\boldsymbol{\mu}_{3}$. Moreover, this group is the unique balanced semidirect product of $\boldsymbol{\mu}_{p}$ and $\boldsymbol{\mu}_{3}$.

Example 2.4. Let $p_{1}$ and $p_{2}$ be distinct prime numbers congruent to 1 modulo 3 , and let $G_{p_{1}} \cong \boldsymbol{\mu}_{p_{1}} \rtimes \boldsymbol{\mu}_{3}$ be the balanced semidirect product. Then the group $G_{p_{1}} \times \boldsymbol{\mu}_{p_{2}}$ is isomorphic to a semidirect product $\boldsymbol{\mu}_{p_{1} p_{2}} \rtimes \boldsymbol{\mu}_{3}$ which is not balanced. Indeed, the image of the corresponding homomorphism

$$
\chi: \boldsymbol{\mu}_{3} \rightarrow \boldsymbol{\mu}_{p_{1} p_{2}}^{*} \cong \boldsymbol{\mu}_{p_{1}-1} \times \boldsymbol{\mu}_{p_{2}-1}
$$

is contained in the subgroup $\boldsymbol{\mu}_{p_{1} p_{2}}\left(p_{1}\right)^{*} \cong \boldsymbol{\mu}_{p_{1}-1}$ of $\boldsymbol{\mu}_{p_{1} p_{2}}^{*}$.

We point out that for a given positive integer $n$ a balanced semidirect product of order $3 n$ may be not unique. 
Example 2.5. Let $p_{1}$ and $p_{2}$ be distinct prime numbers congruent to 1 modulo 3 , and let $n=p_{1} p_{2}$. Then $\boldsymbol{\mu}_{n}^{*} \cong \boldsymbol{\mu}_{p_{1}-1} \times \boldsymbol{\mu}_{p_{2}-1}$, and each of the (cyclic) groups $\boldsymbol{\mu}_{n}\left(p_{i}\right)^{*} \cong \boldsymbol{\mu}_{p_{i}-1}$ contains a unique subgroup of order 3 . Let $\delta_{i}, i=1,2$, be generators of these subgroups. Set $d_{1}=\delta_{1} \delta_{2}$ and $d_{2}=\delta_{1} \delta_{2}^{-1}$. Let $\chi_{1}, \chi_{2}: \boldsymbol{\mu}_{3} \rightarrow \boldsymbol{\mu}_{n}^{*}$ be the homomorphisms that send a generator of $\boldsymbol{\mu}_{3}$ to $d_{1}$ and $d_{2}$, respectively. Construct the groups $G_{1}$ and $G_{2}$ as semidirect products of $\boldsymbol{\mu}_{n}$ and $\boldsymbol{\mu}_{3}$ corresponding to the homomorphisms $\chi_{1}$ and $\chi_{2}$, respectively. We claim that $G_{1}$ is not isomorphic to $G_{2}$. Indeed, while it is easy to construct an automorphism $\zeta$ of $\boldsymbol{\mu}_{n}^{*}$ such that $\chi_{2}=\zeta \circ \chi_{1}$, there does not exist an inner automorphism $\zeta$ of $\boldsymbol{\mu}_{n}^{*}$ like this (that is, the automorphism $\zeta$ cannot be obtained as a conjugation with an element of $\boldsymbol{\mu}_{n}^{*}$ ), because the group $\boldsymbol{\mu}_{n}^{*}$ is abelian. Therefore, the groups $G_{1}$ and $G_{2}$ are not isomorphic to each other by [BE99, Theorem 5.1].

The next result shows that all non-balanced semidirect products have a structure similar to what we see in Example 2.4.

Lemma 2.6. Let $n$ be a positive integer divisible only by primes congruent to 1 modulo 3, let $\chi: \boldsymbol{\mu}_{3} \rightarrow \boldsymbol{\mu}_{n}^{*}$ be a homomorphism, and let $G$ be a semidirect product of $\boldsymbol{\mu}_{n}$ and $\boldsymbol{\mu}_{3}$ corresponding to $\chi$. Then $\chi$ is balanced if and only if the only element of $\boldsymbol{\mu}_{n}$ that commutes with the subgroup $\boldsymbol{\mu}_{3}$ is trivial. Moreover, if $\chi$ is not balanced, then there exists an isomorphism

$$
G \cong\left(\boldsymbol{\mu}_{n_{1}} \rtimes \boldsymbol{\mu}_{3}\right) \times \boldsymbol{\mu}_{n_{2}},
$$

where $n_{1} n_{2}=n$ and $n_{2}>1$.

Proof. Suppose that $\chi$ is not balanced. Then there exists a prime divisor $p$ of $n$ such that the composition of $\chi$ with the projection $\boldsymbol{\mu}_{n}^{*} \rightarrow \boldsymbol{\mu}_{n}(p)^{*}$ is trivial. Hence, the group $\boldsymbol{\mu}_{3}$ commutes with the $p$-Sylow subgroup $\boldsymbol{\mu}_{n}(p) \cong p^{r}$ of $\boldsymbol{\mu}_{n}$. Therefore, we get a required isomorphism $G \cong\left(\boldsymbol{\mu}_{n_{1}} \rtimes \boldsymbol{\mu}_{3}\right) \times \boldsymbol{\mu}_{n_{2}}$, where $n_{1}=\frac{n}{p^{r}}$ and $n_{2}=p^{r}$.

Conversely, suppose that $\chi$ is balanced. Denote by $d \in \boldsymbol{\mu}_{n}^{*}$ the image of a generator of $\boldsymbol{\mu}_{3}$ with respect to $\chi$, so that $d^{3} \equiv 1(\bmod n)$. Assume that there exists a non-trivial element $t \in \boldsymbol{\mu}_{n}$ that commutes with $\boldsymbol{\mu}_{3}$. Considering $\boldsymbol{\mu}_{n}$ as the group of residues modulo $n$, we can write

$$
t d \equiv t \quad(\bmod n)
$$

Since $\boldsymbol{\mu}_{n}$ is isomorphic to a direct product of its Sylow subgroups, we conclude that there is a prime divisor $p$ of $n$ such that the image $t_{p}$ of $t$ in the $p$-Sylow subgroup $\boldsymbol{\mu}_{n}(p) \cong \boldsymbol{\mu}_{p^{r}}$ of $\boldsymbol{\mu}_{n}$ is non-trivial. We have

$$
t_{p} d \equiv t_{p} \quad\left(\bmod p^{r}\right)
$$

Also, we know that $d \not \equiv 1\left(\bmod p^{r}\right)$ because the homomorphism $\chi$ is balanced, and $d^{3} \equiv 1\left(\bmod p^{r}\right)$.

We see from $(2.2)$ that $d \equiv 1(\bmod p)$. Let $s$ be the maximal integer such that $d \equiv 1\left(\bmod p^{s}\right)$; then $1 \leqslant s<r$. Write

$$
d \equiv c p^{s}+1 \quad\left(\bmod p^{s+1}\right),
$$

where $c \not \equiv 0(\bmod p)$. One has

$$
1 \equiv d^{3} \equiv 3 c p^{s}+1 \quad\left(\bmod p^{s+1}\right) .
$$

Since the numbers 3 and $c$ are not divisible by $p$, this gives a contradiction. 
Remark 2.7. In the notation of Lemma 2.6, one can choose $n_{1}$ and $n_{2}$ so that the semidirect product $\boldsymbol{\mu}_{n_{1}} \rtimes \boldsymbol{\mu}_{3}$ in (2.1) is balanced.

We conclude this section by two simple general observations concerning semidirect products.

Lemma 2.8. Let $n$ and $m$ be positive integers, and let $d$ be an integer such that $d^{m} \equiv 1(\bmod n)$. Let $H$ be a group generated by an element $x$ of order $n$ and an element $y$ of order $m$ subject to relation

$$
x y=y x^{d} .
$$

Suppose that the cyclic subgroups generated by $x$ and $y$ have trivial intersection. Then $H \cong \boldsymbol{\mu}_{n} \rtimes \boldsymbol{\mu}_{m}$, and the semidirect product structure corresponds to the homomorphism $\boldsymbol{\mu}_{m} \rightarrow \boldsymbol{\mu}_{n}^{*}$ sending a generator of $\boldsymbol{\mu}_{m}$ to $d \in \boldsymbol{\mu}_{n}^{*}$.

Proof. We see from relation (2.3) that every element of $H$ can be written in the form $y^{r} x^{s}$ for some positive integers $r$ and $s$. Therefore, $H$ is a quotient of the semidirect product $\tilde{H} \cong \boldsymbol{\mu}_{n} \rtimes \boldsymbol{\mu}_{m}$ corresponding to the homomorphism $\boldsymbol{\mu}_{m} \rightarrow \boldsymbol{\mu}_{n}^{*}$ that sends a generator of $\boldsymbol{\mu}_{m}$ to $d \in \boldsymbol{\mu}_{n}^{*}$. On the other hand, if for some $r_{1}, r_{2}, s_{1}$, and $s_{2}$ one has $y^{r_{1}} x^{s_{1}}=y^{r_{2}} x^{s_{2}}$, then $y^{r_{1}-r_{2}}=x^{s_{2}-s_{1}}$. Since the cyclic subgroups generated by $x$ and $y$ have trivial intersection, this implies that $r_{1} \equiv r_{2}(\bmod m)$ and $s_{1} \equiv s_{2}(\bmod n)$. Hence $|H|=m n=|\tilde{H}|$, which means that $H \cong \tilde{H}$.

Lemma 2.9. Let $G$ be a finite group that does not contain elements of order 9 , and let $H$ be a normal subgroup of index 3 in $G$. Then $G \cong H \rtimes \boldsymbol{\mu}_{3}$.

Proof. Choose a preimage $g$ of a generator of the group $G / H \cong \boldsymbol{\mu}_{3}$ with respect to the projection $\psi: G \rightarrow G / H$. The order of $g$ is divisible by 3 , but is not divisible by 9 . Thus it equals $3 m$ for some positive integer $m$ not divisible by 3 . Hence the element $g^{\prime}=g^{m}$ has order 3 , and $\psi\left(g^{\prime}\right)$ generates $G / H$. This means that the subgroup $F \cong \boldsymbol{\mu}_{3}$ in $G$ generated by $g^{\prime}$ has trivial intersection with $H$, and thus $G$ is a semidirect product of $H$ and $F$.

We remark that one cannot drop the assumption about the absence of elements of order 9 in Lemma 2.9. Indeed, the group $\boldsymbol{\mu}_{9}$ contains a normal subgroup $\boldsymbol{\mu}_{3}$ of index 3, but it is not isomorphic to a (semi)direct product of two copies of $\boldsymbol{\mu}_{3}$. Note also that under a stronger assumption that $H$ does not contain elements of order 3 the assertion of Lemma 2.9 becomes a particular case of a much more general Schur-Zassenhaus theorem, see e.g. [Isa08, Theorem 3.5].

\section{Central simple Algebras}

In this section we gather auxiliary facts concerning Severi-Brauer surfaces, and also some assertions from Galois theory.

We refer the reader to [Art82] and Kol16] for the basic theory of Severi-Brauer surfaces and higher-dimensional Severi-Brauer varieties. The foundational fact of this theory is that Severi-Brauer varieties of dimension $m$ over a field $\mathbb{K}$ are in one-to-one correspondence with central simple algebras of dimension $(m+1)^{2}$ over $\mathbb{K}$, with $\mathbb{P}^{m}$ corresponding to the algebra of $(m+1) \times(m+1)$-matrices. A Severi-Brauer variety over $\mathbb{K}$ is trivial if and only if it has a $\mathbb{K}$-point.

Given an algebra $A$, we denote by $A^{*}$ the multiplicative group of its invertible elements. The following characterization of the automorphism group of a Severi-Brauer variety is well-known, see Theorem E on page 266 of [Châ44], or [ShV20, Lemma 4.1]. 
Lemma 3.1. Let $X$ be a Severi-Brauer variety over a field $\mathbb{K}$ corresponding to a central simple algebra $A$. Then $\operatorname{Aut}(X) \cong A^{*}(\mathbb{K}) / \mathbb{K}^{*}$.

Let $\mathbb{L} / \mathbb{K}$ be a Galois extension with Galois group isomorphic to $\boldsymbol{\mu}_{3}$. Choose an element $a \in \mathbb{K}^{*}$ and a generator $\sigma$ of $\operatorname{Gal}(\mathbb{L} / \mathbb{K})$. Then one can associate with $\sigma$ and $a$ a cyclic algebra $A$, which is a central simple algebra over $\mathbb{K}$, see [GS06, §2.5] or [GSh18, Exercise 3.1.6]. Explicitly, $A$ is generated over $\mathbb{K}$ by $\mathbb{L}$ and an element $\alpha$ subject to relations $\alpha^{3}=a$ and

$$
\lambda \alpha=\alpha \sigma(\lambda), \quad \lambda \in \mathbb{L} .
$$

Lemma 3.2 (see e.g. [GSh18, Exercise 3.1.6(i)]). Suppose that in the above notation the element $a$ is not contained in the image of the Galois norm $N_{\mathbb{L} / \mathbb{K}}$. Then $A$ is not isomorphic to a matrix algebra.

It appears that the above construction is responsible for all possible central simple algebras of dimension 9 over an arbitrary field $\mathbb{K}$ (with a trivial exception of the situation when $\mathbb{K}$ has no cyclic extensions of degree 3 , in which case there are no central simple algebras of dimension 9 over $\mathbb{K}$ apart from the matrix algebra anyway). Namely, the following result can be found in [Wed21] or [GS06, Chapter 7, Exercise 9].

Lemma 3.3. Let $A$ be a central simple algebra of dimension 9 over a field $\mathbb{K}$. Suppose that $A$ is not isomorphic to the matrix algebra. Then $A$ is a cyclic algebra constructed from some cyclic Galois extension $\mathbb{L} / \mathbb{K}$ and some element $a \in \mathbb{K}^{*}$.

The next fact is immediately implied by the theorem of Wedderburn about the structure of central simple algebras, see [GS06, Theorem 2.1.3].

Lemma 3.4. Let $A$ be a central simple algebra of dimension $q^{2}$ over a field $\mathbb{K}$, where $q$ is a prime number. Then $A$ is either the matrix algebra, or a division algebra.

We will also need the following facts from Galois theory.

Theorem 3.5 ([CF67, $\S$ III.1]). Let $n$ be a positive integer, and let $\xi$ be a primitive $n$-th root of unity. Then $\mathbb{Q}(\xi) / \mathbb{Q}$ is a Galois extension, and

$$
\operatorname{Gal}(\mathbb{Q}(\xi) / \mathbb{Q}) \cong \boldsymbol{\mu}_{n}^{*}
$$

Lemma 3.6. Let $\xi_{1}$ and $\xi_{2}$ be primitive roots of unity of degrees $n_{1}$ and $n_{2}$, respectively. Suppose that $n_{1}$ and $n_{2}$ are coprime, and set $\mathbb{M}=\mathbb{Q}\left(\xi_{1}\right)$. Then $\mathbb{M}\left(\xi_{2}\right) / \mathbb{M}$ is a Galois extension, and

$$
\operatorname{Gal}\left(\mathbb{M}\left(\xi_{2}\right) / \mathbb{M}\right) \cong \boldsymbol{\mu}_{n_{2}}^{*}
$$

Proof. Note that $\mathbb{Q}\left(\xi_{1}\right) \cap \mathbb{Q}\left(\xi_{2}\right)=\mathbb{Q}$, see for instance [Lan65, §VIII.3]. Also, we know from Theorem 3.5 that $\mathbb{Q}\left(\xi_{2}\right) / \mathbb{Q}$ is a Galois extension, and

$$
\operatorname{Gal}\left(\mathbb{Q}\left(\xi_{2}\right) / \mathbb{Q}\right) \cong \boldsymbol{\mu}_{n_{2}}^{*}
$$

Thus the assertion follows from [Lan65, Theorem VIII.1.4].

The next result is well known to experts; it follows for instance from [Ste89, Theorem 1(b)].

Lemma 3.7. Let $\mathbb{L} / \mathbb{K}$ be a finite Galois extension of number fields. Then the Galois norm map $N_{\mathbb{L} / \mathbb{K}}: \mathbb{L}^{*} \rightarrow \mathbb{K}^{*}$ is not surjective. 


\section{Construction of subgroups}

In this section we construct examples of finite groups acting on non-trivial SeveriBrauer surfaces. It is easy to produce a non-trivial Severi-Brauer surface with an action of the group $\boldsymbol{\mu}_{3}^{2}$.

Example 4.1 (see [Sh20a, Example 4.7]). Let $\mathbb{K}$ be a field of characteristic different from 3 that contains a non-trivial cubic root of unity $\omega$. Let $a$ and $b$ be elements of $\mathbb{K}$ such that $b$ is not a cube in $\mathbb{K}$, and $a$ is not contained in the image of the Galois norm for the field extension $\mathbb{L} / \mathbb{K}$, where

$$
\mathbb{L}=\mathbb{K}(\sqrt[3]{b}) .
$$

If $\mathbb{K}$ is a number field, then an element $b$ like this always exists for obvious reasons, and an element $a$ exists by Lemma 3.7. Consider the algebra $A$ over $\mathbb{K}$ generated by variables $u$ and $v$ subject to relations

$$
u^{3}=a, \quad v^{3}=b, \quad u v=\omega v u .
$$

Then $A$ is a central simple algebra, and moreover a division algebra; actually, one can construct it as a cyclic algebra over $\mathbb{K}$ associated with (a generator of the Galois group of) the cyclic extension $\mathbb{L} / \mathbb{K}$ and the element $a$. Thus $A$ corresponds to a non-trivial SeveriBrauer surface $S$. One can see that $u$ and $v$ generate a non-abelian subgroup of order 27 and exponent 3 in $A^{*}$ (sometimes called the Heisenberg group). Their images in $A^{*} / \mathbb{K}^{*}$ generate a group isomorphic to $\boldsymbol{\mu}_{3}^{2}$, which is embedded as a subgroup into $\operatorname{Aut}(S)$ by Lemma 3.1.

Remark 4.2. Let $S$ be a Severi-Brauer surface over a field $\mathbb{K}$. Then $S$ has an automorphism of order 3. Indeed, if $S \cong \mathbb{P}^{2}$, then this is obvious. Otherwise it follows from Lemma 3.3 that $S$ corresponds to some cyclic algebra. In the notation of $\$ 3$, the algebra $A$ contains the element $\alpha \notin \mathbb{K}$ such that $\alpha^{3} \in \mathbb{K}$. Thus $\alpha$ defines an automorphism of $S$ of order 3 by Lemma 3.1.

The proof of the next result is similar to that of Theorem 1.2, see [Sh20b].

Lemma 4.3. Let $n>1$ be an integer divisible only by primes congruent to 1 modulo 3 . Let $G \cong \boldsymbol{\mu}_{n} \rtimes \boldsymbol{\mu}_{3}$ be a balanced semidirect product. Then there exists a number field $\mathbb{K}$ and a non-trivial Severi-Brauer surface $S$ over $\mathbb{K}$ such that the group $\operatorname{Aut}(S)$ contains $G$.

Proof. Let $\xi$ denote a primitive root of unity of degree $n$, and let $\mathbb{L}=\mathbb{Q}(\xi)$. Then $\mathbb{L} / \mathbb{Q}$ is a Galois extension with the Galois group isomorphic to $\boldsymbol{\mu}_{n}^{*}$ by Theorem 3.5. Let $\chi: \boldsymbol{\mu}_{3} \rightarrow \boldsymbol{\mu}_{n}^{*}$ be the homomorphism giving rise to the semidirect product structure on $G$. Consider $\chi$ as a homomorphism

$$
\chi: \boldsymbol{\mu}_{3} \rightarrow \operatorname{Gal}(\mathbb{L} / \mathbb{Q}),
$$

and let $\mathbb{K} \subset \mathbb{L}$ be the subfield of invariants of the subgroup $\chi\left(\boldsymbol{\mu}_{3}\right) \subset \operatorname{Gal}(\mathbb{L} / \mathbb{Q})$. Then $\mathbb{L} / \mathbb{K}$ is a Galois extension with the Galois group $\mathrm{Gal}(\mathbb{L} / \mathbb{K})$ isomorphic to $\boldsymbol{\mu}_{3}$. Let $\sigma$ be a generator of $\mathrm{Gal}(\mathbb{L} / \mathbb{K})$. Since $\chi$ is balanced, we conclude from Lemma 2.6 that for all $1 \leqslant m<n$ one has $\sigma\left(\xi^{m}\right) \neq \xi^{m}$; thus $\xi^{m} \notin \mathbb{K}$. Explicitly, $\sigma$ sends $\xi \in \mathbb{L}$ to $\xi^{d}$, where $d \in \boldsymbol{\mu}_{n}^{*}$ is the image of a generator of $\boldsymbol{\mu}_{3}$ under the embedding $\chi: \boldsymbol{\mu}_{3} \rightarrow \boldsymbol{\mu}_{n}^{*}$. In particular, one has $d^{3} \equiv 1(\bmod n)$.

By Lemma 3.7 there exists an element $a \in \mathbb{K}$ such that $a$ is not contained in the image of the Galois norm of the field extension $\mathbb{L} / \mathbb{K}$. Consider the cyclic algebra $A$ over $\mathbb{K}$ 
associated with $\sigma$ and $a$. We know that $A$ is a central simple algebra. However, $A$ is not a matrix algebra by Lemma 3.2. Since the dimension of $A$ over $\mathbb{K}$ equals 9 , we conclude that $A$ is a division algebra by Lemma 3.4. The algebra $A$ contains the root of unity $\xi \in \mathbb{L}$ and an element $\alpha$ such that $\alpha^{3}=a$ and relation

$$
\xi \alpha=\alpha \xi^{d}
$$

holds.

Denote by $\hat{\alpha}$ and $\hat{\xi}$ the images in the quotient group $A^{*} / \mathbb{K}^{*}$ of the elements $\alpha$ and $\xi$, respectively, and denote by $\hat{G}$ the subgroup of $A^{*} / \mathbb{K}^{*}$ generated by $\hat{\alpha}$ and $\hat{\xi}$. Since $\alpha^{3} \in \mathbb{K}$ and $\alpha \notin \mathbb{K}$, the order of $\hat{\alpha}$ in $A^{*} / \mathbb{K}^{*}$ equals 3 as well. Similarly, one has $\xi^{n}=1 \in \mathbb{K}$, and $\xi^{m} \notin \mathbb{K}$ for all $1 \leqslant m<n$. Thus the order of $\hat{\xi}$ in $A^{*} / \mathbb{K}^{*}$ equals $n$. Since $n$ is coprime to 3 , the cyclic groups generated by $\hat{\xi}$ and $\hat{\alpha}$ have trivial intersection. Furthermore, relation (4.1) implies

$$
\hat{\xi} \hat{\alpha}=\hat{\alpha} \hat{\xi}^{d}
$$

Hence $\hat{G}$ is the semidirect product of $\boldsymbol{\mu}_{n}$ and $\boldsymbol{\mu}_{3}$ corresponding to $\chi$ by Lemma 2.8, so that $\hat{G} \cong G$.

We see that the elements $\hat{\xi}$ and $\hat{\alpha}$ generate a subgroup isomorphic to $G$ in $A^{*} / \mathbb{K}^{*}$. On the other hand, for a Severi-Brauer surface $S$ corresponding to $A$ one has $\operatorname{Aut}(S) \cong A^{*} / \mathbb{K}^{*}$ by Lemma 3.1 .

Remark 4.4. If in the notation of the proof of Lemma 4.3 we choose $\chi: \boldsymbol{\mu}_{3} \rightarrow \mathrm{Gal}(\mathbb{L} / \mathbb{Q})$ to be a non-balanced homomorphism, and set $\mathbb{K}$ to be the field of invariants of the group $\chi\left(\boldsymbol{\mu}_{3}\right)$, then for some $1 \leqslant m<n$ the element $\xi^{m}$ is invariant under the action of the Galois group $\operatorname{Gal}(\mathbb{L} / \mathbb{K})$. This means that $\xi^{m}$ is an element of $\mathbb{K}$. Therefore, while the subgroup of $A^{*}$ generated by $\xi$ and $\alpha$ has a quotient isomorphic to the non-balanced semidirect product $\boldsymbol{\mu}_{n} \rtimes \boldsymbol{\mu}_{3}$ corresponding to $\chi$, its image in $\operatorname{Aut}(S) \cong A^{*} / \mathbb{K}^{*}$ is isomorphic to a (balanced) semidirect product $\boldsymbol{\mu}_{n^{\prime}} \rtimes \boldsymbol{\mu}_{3}$ for some $n^{\prime}<n$, cf. Remark 2.7.

The following result is proved by a blend of constructions used in Example 4.1 and Lemma 4.3.

Lemma 4.5. Let $n \geqslant 1$ be an integer divisible only by primes congruent to 1 modulo 3 . Let $G^{\prime} \cong \boldsymbol{\mu}_{n} \rtimes \boldsymbol{\mu}_{3}$ be a balanced semidirect product, and let $G \cong \boldsymbol{\mu}_{3} \times G^{\prime}$. Then there exists a number field $\mathbb{K}$ and a non-trivial Severi-Brauer surface $S$ over $\mathbb{K}$ such that the group $\operatorname{Aut}(S)$ contains $G$.

Proof. Let $\omega$ and $\xi$ denote primitive roots of unity of degrees 3 and $n$, respectively; let $\tau$ be a cubic root of 2 . Set $\mathbb{M}=\mathbb{Q}(\omega)$ and $\mathbb{L}=\mathbb{M}(\xi, \tau)$. We observe that

$$
\mathbb{M}(\tau) \cap \mathbb{M}(\xi)=\mathbb{M} \text {. }
$$

Indeed, this intersection contains $\mathbb{M}$ and is contained in $\mathbb{M}(\tau)$. The degree of the extension $\mathbb{M}(\tau) / \mathbb{M}$ equals 3 , so we only need to check that $\mathbb{M}(\tau)$ is not contained in $\mathbb{M}(\xi)$. On the other hand, if $\mathbb{M}(\tau) \subset \mathbb{M}(\xi)$, then $\mathbb{Q}(\tau)$ is a subfield of $\mathbb{M}(\xi)$ as well. Since

$$
\mathbb{M}(\xi)=\mathbb{Q}(\omega, \xi)=\mathbb{Q}(\omega \xi),
$$

we know from Theorem 3.5 that the Galois group $\operatorname{Gal}(\mathbb{M}(\xi) / \mathbb{Q})$ is cyclic. Hence every subfield of $\mathbb{M}(\xi)$ is normal over $\mathbb{Q}$, which is not the case for $\mathbb{Q}(\tau)$.

Note that $\mathbb{M}(\xi) / \mathbb{M}$ is a Galois extension with Galois group isomorphic to $\boldsymbol{\mu}_{n}^{*}$ by Lemma 3.6, and $\mathbb{M}(\tau) / \mathbb{M}$ is a Galois extension with Galois group isomorphic to $\boldsymbol{\mu}_{3}$. 
Since the extension $\mathbb{L} / \mathbb{M}$ can be represented as the composite of the extensions $\mathbb{M}(\tau) / \mathbb{M}$ and $\mathbb{M}(\xi) / \mathbb{M}$, we conclude that

$$
\operatorname{Gal}(\mathbb{L} / \mathbb{M}) \cong \operatorname{Gal}(\mathbb{M}(\tau) / \mathbb{M}) \times \operatorname{Gal}(\mathbb{M}(\xi) / \mathbb{M}) \cong \boldsymbol{\mu}_{3} \times \boldsymbol{\mu}_{n}^{*},
$$

see for instance [Lan65, Theorem VIII.1.5].

Let $\chi: \boldsymbol{\mu}_{3} \rightarrow \operatorname{Gal}(\mathbb{L} / \mathbb{M})$ be the homomorphism such that its composition with the projection $\operatorname{Gal}(\mathbb{L} / \mathbb{M}) \rightarrow \operatorname{Gal}(\mathbb{M}(\tau) / \mathbb{M})$ is an isomorphism $\boldsymbol{\mu}_{3} \stackrel{\sim}{\longrightarrow} \boldsymbol{\mu}_{3}$, and the composition with the projection $\operatorname{Gal}(\mathbb{L} / \mathbb{M}) \rightarrow \operatorname{Gal}(\mathbb{M}(\xi) / \mathbb{M})$ is the homomorphism

$$
\chi^{\prime}: \boldsymbol{\mu}_{3} \rightarrow \boldsymbol{\mu}_{n}^{*}
$$

giving rise to the semidirect product structure on $G^{\prime}$. Let $\mathbb{K} \subset \mathbb{L}$ be the subfield of invariants of the subgroup $\chi\left(\boldsymbol{\mu}_{3}\right) \subset \mathrm{Gal}(\mathbb{L} / \mathbb{M})$. Then $\mathbb{L} / \mathbb{K}$ is a Galois extension such that $\operatorname{Gal}(\mathbb{L} / \mathbb{K}) \cong \boldsymbol{\mu}_{3}$. A generator $\sigma$ of $\operatorname{Gal}(\mathbb{L} / \mathbb{K})$ sends $\xi \in \mathbb{L}$ to $\xi^{d}$, where $d$ is some integer such that $d^{3} \equiv 1(\bmod n)$, and sends $\tau \in \mathbb{L}$ to $\omega \tau$. Since the homomorphism $\chi^{\prime}$ is balanced, we conclude from Lemma 2.6 that for all $1 \leqslant m<n$ one has $\sigma\left(\xi^{m}\right) \neq \xi^{m}$; thus $\xi^{m} \notin \mathbb{K}$.

By Lemma 3.7 there exists an element $a \in \mathbb{K}$ such that $a$ is not contained in the image of the Galois norm of the field extension $\mathbb{L} / \mathbb{K}$. Consider the cyclic algebra $A$ over $\mathbb{K}$ associated with $\sigma$ and $a$. We know that $A$ is a central simple algebra, and $A$ is not a matrix algebra by Lemma 3.2. Thus $A$ is a division algebra by Lemma 3.4. The algebra $A$ contains the $n$-th root of unity $\xi$ and an element $\alpha$ such that $\alpha^{3}=a$ and relation (4.1). holds. Furthermore, $A$ contains $\tau$, and

$$
\tau \alpha=\omega \alpha \tau .
$$

Denote by $\hat{\alpha}, \hat{\xi}$, and $\hat{\tau}$ the images in the quotient group $A^{*} / \mathbb{K}^{*}$ of the elements $\alpha, \xi$, and $\tau$, respectively. Denote by $\hat{G}^{\prime}$ the subgroup of $A^{*} / \mathbb{K}^{*}$ generated by $\hat{\alpha}$ and $\hat{\xi}$, and by $\hat{G}$ the subgroup generated by $\hat{\alpha}, \hat{\xi}$, and $\hat{\tau}$. The order of $\hat{\alpha}$ in $A^{*} / \mathbb{K}^{*}$ equals 3 . The order of $\hat{\xi}$ in $A^{*} / \mathbb{K}^{*}$ equals $n$, and the cyclic groups generated by $\hat{\xi}$ and $\hat{\alpha}$ have trivial intersection. Using relation (4.1) and Lemma 2.8, we see that $\hat{G}^{\prime}$ is the semidirect product of $\boldsymbol{\mu}_{n}$ and $\boldsymbol{\mu}_{3}$ corresponding to the homomorphism $\chi^{\prime}$, and so $\hat{G}^{\prime} \cong G^{\prime}$ (cf. the proof of Lemma 4.3).

Since $\omega \in \mathbb{K}$, we see from relation (4.2) that the group $\hat{G}^{\prime \prime}$ generated by $\hat{\alpha}$ and $\hat{\tau}$ is isomorphic to $\boldsymbol{\mu}_{3}^{2}$ (cf. Example 4.1). In particular, $\hat{\tau}$ has order 3 and commutes with $\hat{\alpha}$. Since $\tau$ and $\xi$ are elements of the field $\mathbb{L} \subset A$, they commute with each other, and thus $\hat{\tau}$ commutes with $\hat{\xi}$. Hence $\hat{\tau}$ commutes with the group $\hat{G}^{\prime}$ generated by $\hat{\alpha}$ and $\hat{\xi}$. Since $n$ is coprime to 3 , the group $\hat{G}^{\prime \prime}$ has trivial intersection with the cyclic group generated by $\hat{\xi}$. This implies that the cyclic group $\boldsymbol{\mu}_{3}$ generated by $\hat{\tau}$ has trivial intersection with the group $\hat{G}^{\prime}$. Thus we conclude that

$$
\hat{G} \cong \boldsymbol{\mu}_{3} \times \hat{G}^{\prime} \cong \boldsymbol{\mu}_{3} \times G^{\prime} \cong G .
$$

Therefore, the elements $\hat{\xi}, \hat{\alpha}$, and $\hat{\tau}$ generate a subgroup isomorphic to $G$ in $A^{*} / \mathbb{K}^{*}$. Thus the automorphism group of the Severi-Brauer surface corresponding to $A$ contains $G$ by Lemma 3.1.

Lemmas 4.3 and 4.5 imply the following.

Corollary 4.6. Let $n=3^{r} \prod p_{i}^{r_{i}}$ be a positive integer, where $p_{i}$ are primes congruent to 1 modulo 3 , and $r \leqslant 1$. Then there exists a number field $\mathbb{K}$ and a non-trivial Severi-Brauer surface $S$ over $\mathbb{K}$ such that the group $\operatorname{Aut}(S)$ contains an element of order $n$. 


\section{Automorphisms of PRIME ORDER}

In this section we describe the possible finite orders of automorphisms of non-trivial Severi-Brauer surfaces.

Corollary 4.6 shows that for every positive integer $n$ not divisible by 9 and not divisible by primes congruent to 2 modulo 3 there exists a non-trivial Severi-Brauer surface over a field of characteristic zero with an automorphism of order $n$. We complete this picture by showing that these are all possible finite orders of automorphisms of non-trivial SeveriBrauer surfaces. Let us start with a general observation.

Lemma 5.1. Let $S$ be a non-trivial Severi-Brauer surface over a field $\mathbb{K}$ of characteristic zero. Let $g$ be a non-trivial automorphism of finite order of $S$. Then $g$ has exactly three fixed points on $S_{\overline{\mathbb{K}}}$, and these points are transitively permuted by the Galois group $\mathrm{Gal}(\overline{\mathbb{K}} / \mathbb{K})$. In particular, g cannot have a unique isolated fixed point on $S_{\overline{\mathbb{K}}}$.

Proof. Since $g$ has finite order, it either has exactly three fixed points on $S_{\overline{\mathbb{K}}}$, or it acts on $S_{\overline{\mathbb{K}}}$ with a unique isolated fixed point. Suppose that the latter is the case. Since the action of $\mathrm{Gal}(\overline{\mathbb{K}} / \mathbb{K})$ on $S$ commutes with the action of $g$, the isolated fixed point must be $\operatorname{Gal}(\overline{\mathbb{K}} / \mathbb{K})$-invariant, and thus defined over $\mathbb{K}$. This is impossible because the SeveriBrauer surface $S$ is non-trivial. Therefore, $g$ has exactly three fixed points on $S_{\overline{\mathbb{K}}}$; since $S$ has no points over $\mathbb{K}$, these three points have to be transitively permuted by Gal $(\overline{\mathbb{K}} / \mathbb{K})$.

Lemma 5.2 (cf. [Sh20a, Lemma 4.2]). Let $p \equiv 2(\bmod 3)$ be a prime number. Let $S$ be a Severi-Brauer surface over a field $\mathbb{K}$ of characteristic zero such that the group Aut $(S)$ contains an element $g$ of order $p$. Then $S \cong \mathbb{P}^{2}$.

Proof. Suppose that the Severi-Brauer surface $S$ is non-trivial, and consider the action of $g$ on $S_{\overline{\mathbb{K}}} \cong \mathbb{P}_{\overline{\mathbb{K}}}^{2}$. Let $\tilde{g}$ be a preimage of $g$ under the natural projection

$$
\pi: \mathrm{SL}_{3}(\overline{\mathbb{K}}) \rightarrow \operatorname{Aut}\left(\mathbb{P}_{\overline{\mathbb{K}}}^{2}\right) \cong \mathrm{PGL}_{3}(\overline{\mathbb{K}})
$$

The order of $\tilde{g}$ equals either $p$ or $3 p$. Since $p$ is not divisible by 3 , we can multiply $\tilde{g}$ by an appropriate scalar matrix so that the order of $\tilde{g}$ equals $p$. Moreover, the element $\tilde{g}$ of order $p$ such that $\pi(\tilde{g})=g$ is unique in $\mathrm{SL}_{3}(\overline{\mathbb{K}})$, and hence it is defined over the field $\mathbb{K}$.

Let $\xi_{1}, \xi_{2}$, and $\xi_{3}$ be the eigen-values of $\tilde{g}$; these are $p$-th roots of unity. According to Lemma [5.1, the element $g$ has exactly three fixed points on $\mathbb{P}_{\mathbb{K}}^{2}$, and these points are transitively permuted by the group $\mathrm{Gal}(\overline{\mathbb{K}} / \mathbb{K})$. In other words, the roots of unity $\xi_{1}, \xi_{2}$, and $\xi_{3}$ are pairwise different and form a Gal $(\overline{\mathbb{K}} / \mathbb{K})$-orbit. Hence the image $\Gamma$ of $\mathrm{Gal}(\overline{\mathbb{K}} / \mathbb{K})$ in the automorphism group $\operatorname{Aut}\left(\boldsymbol{\mu}_{p}\right) \cong \boldsymbol{\mu}_{p-1}$ of the multiplicative group $\boldsymbol{\mu}_{p}$ of $p$-th roots of unity has an orbit of order 3 . This means that the order of $\Gamma$ is divisible by 3 . However, the number $p-1$ is not divisible by 3 by assumption, which gives a contradiction.

The argument from the proof of Lemma 5.2 cannot be applied to an automorphism of a Severi-Brauer surface whose order is divisible by 3, since in this case we cannot always find a preimage of such an automorphism in $\mathrm{SL}_{3}(\overline{\mathbb{K}})$ defined over the base field $\mathbb{K}$. However, we can study such elements using a slightly modified argument.

Lemma 5.3. Let $S$ be a Severi-Brauer surface over a field $\mathbb{K}$ of characteristic zero such that the group $\operatorname{Aut}(S)$ contains an element $g$ of order 9 . Then $S \cong \mathbb{P}^{2}$. 
Proof. Suppose that the Severi-Brauer surface $S$ is non-trivial. According to Lemma 5.1, the element $g$ has exactly three fixed points $P_{1}, P_{2}$, and $P_{3}$ on $\mathbb{P}_{\overline{\mathbb{K}}}^{2}$, and these points are transitively permuted by the group $\mathrm{Gal}(\overline{\mathbb{K}} / \mathbb{K})$. Let $T_{i} \cong \overline{\mathbb{K}}^{2}$ be the Zariski tangent space to $\mathbb{P}_{\overline{\mathbb{K}}}^{2}$ at the point $P_{i}$. Then $g$ acts faithfully on $T_{i}$. Let $\xi_{i}^{\prime}$ and $\xi_{i}^{\prime \prime}$ be the eigen-values of this action. Choose some preimage $\tilde{g}$ of $g$ under the projection

$$
\pi: \mathrm{SL}_{3}(\overline{\mathbb{K}}) \rightarrow \mathrm{PGL}_{3}(\overline{\mathbb{K}})
$$

we emphasize that $\tilde{g}$ may not be defined over $\mathbb{K}$. Let $\xi_{1}, \xi_{2}$, and $\xi_{3}$ be the eigen-values of $\tilde{g}$ corresponding to the fixed points $P_{1}, P_{2}$, and $P_{3}$ of $g$, respectively. Relabelling the numbers $\xi_{i}^{\prime}$ and $\xi_{i}^{\prime \prime}$ if necessary, we may assume that

$$
\xi_{1}^{\prime}=\frac{\xi_{2}}{\xi_{1}}, \quad \xi_{1}^{\prime \prime}=\frac{\xi_{3}}{\xi_{1}}, \quad \xi_{2}^{\prime}=\frac{\xi_{3}}{\xi_{2}}, \quad \xi_{2}^{\prime \prime}=\frac{\xi_{1}}{\xi_{2}}, \quad \xi_{3}^{\prime}=\frac{\xi_{1}}{\xi_{3}}, \quad \xi_{3}^{\prime \prime}=\frac{\xi_{2}}{\xi_{3}} .
$$

Note that $\xi_{i}^{\prime}$ and $\xi_{i}^{\prime \prime}$ are 9-th roots of unity, while $\xi_{i}$ may not be 9-th roots of unity but are always 27 -th roots of unity, because $\tilde{g}^{9}$ is a scalar matrix in $\mathrm{SL}_{3}(\overline{\mathbb{K}})$.

If for some $i \neq j$ we have $\xi_{i}^{3}=\xi_{j}^{3}$, then the non-trivial automorphism $g^{3}$ of $S$ has infinitely many fixed points on $S_{\overline{\mathbb{K}}}$, and thus $S \cong \mathbb{P}^{2}$ by Lemma 5.1. This gives some restrictions on the values of $\xi_{i}^{\prime}$ and $\xi_{i}^{\prime \prime}$. For instance, let $\omega$ be a non-trivial cubic root of unity, let $v$ be one of the numbers $1, \omega$, or $\omega^{2}$. If $\xi_{1}^{\prime}=v \xi_{2}^{\prime \prime}$, then

$$
\frac{\xi_{2}}{\xi_{1}}=v \frac{\xi_{1}}{\xi_{2}}
$$

so that $v \xi_{1}^{2}=\xi_{2}^{2}$. Thus $\xi_{1}^{6}=\xi_{2}^{6}$, and hence $\xi_{1}^{3}=\xi_{2}^{3}$ because both $\xi_{1}$ and $\xi_{2}$ are 27-th roots of unity. Similarly, if $\xi_{1}^{\prime}=v \xi_{3}^{\prime \prime}$, then

$$
\frac{\xi_{2}}{\xi_{1}}=v \frac{\xi_{2}}{\xi_{3}}
$$

and thus $v \xi_{1}=\xi_{3}$ and $\xi_{1}^{3}=\xi_{3}^{3}$. In each of these cases we see that $S \cong \mathbb{P}^{2}$, which contradicts our assumption.

Since the points $P_{1}, P_{2}$, and $P_{3}$ are transitively permuted by the Galois group $\mathrm{Gal}(\overline{\mathbb{K}} / \mathbb{K})$, the action of $\mathrm{Gal}(\overline{\mathbb{K}} / \mathbb{K})$ transitively permutes the non-ordered pairs

$$
\left\{\xi_{1}^{\prime}, \xi_{1}^{\prime \prime}\right\}, \quad\left\{\xi_{2}^{\prime}, \xi_{2}^{\prime \prime}\right\}, \quad\left\{\xi_{3}^{\prime}, \xi_{3}^{\prime \prime}\right\}
$$

(Note that at the same time the collection of eigen-values $\xi_{1}, \xi_{2}, \xi_{3}$ may be not preserved by the Galois group, since $\tilde{g}$ is not necessarily defined over the field $\mathbb{K}$.) Thus there exists an element $\gamma \in \operatorname{Gal}(\overline{\mathbb{K}} / \mathbb{K})$ such that $\gamma\left(\xi_{1}^{\prime}\right)$ and $\gamma^{2}\left(\xi_{1}^{\prime}\right)$ are contained in the second and the third pairs in (5.1), respectively, and $\gamma^{3}\left(\xi_{1}^{\prime}\right)=\xi_{1}^{\prime}$. Such an element defines an automorphism of order 3 of the cyclic subgroup of $\overline{\mathbb{K}}^{*}$ generated by $\xi_{1}^{\prime}$. Since $\xi_{1}^{\prime}$ is a 9-th root of unity, one has $\gamma\left(\xi_{1}^{\prime}\right)=v^{-1} \xi_{1}^{\prime}$ and $\gamma^{2}\left(\xi_{1}^{\prime}\right)=v^{-2} \xi_{1}^{\prime}$, where $v$ is one of the numbers $1, \omega$, or $\omega^{2}$.

The above computation shows that $\gamma\left(\xi_{1}^{\prime}\right) \neq \xi_{2}^{\prime \prime}$ and $\gamma^{2}\left(\xi_{1}^{\prime}\right) \neq \xi_{3}^{\prime \prime}$, so that $\gamma\left(\xi_{1}^{\prime}\right)=\xi_{2}^{\prime}$ and $\gamma^{2}\left(\xi_{1}^{\prime}\right)=\xi_{3}^{\prime}$. In other words, we have

$$
\xi_{1}^{\prime}=v \xi_{2}^{\prime}=v^{2} \xi_{3}^{\prime}
$$

Thus

$$
\frac{\xi_{2}}{\xi_{1}}=v \frac{\xi_{3}}{\xi_{2}}=v^{2} \frac{\xi_{1}}{\xi_{3}}
$$


Hence

$$
\frac{\xi_{2}^{2}}{\xi_{1}}=v \xi_{3}=\frac{\xi_{1}^{2}}{\xi_{2}}
$$

so that $\xi_{1}^{3}=\xi_{2}^{3}$. This again means that $S \cong \mathbb{P}^{2}$, and gives a contradiction.

Let us summarize the results of the last two sections.

Corollary 5.4. Let $n$ be a positive integer. Then there exists a field $\mathbb{K}$ of characteristic zero, a non-trivial Severi-Brauer surface $S$ over $\mathbb{K}$, and an element of order $n$ in the group $\operatorname{Aut}(S)$, if and only if $n=3^{r} \prod p_{i}^{r_{i}}$, where $p_{i}$ are primes congruent to 1 modulo 3 , and $r \leqslant 1$.

Proof. The "if" part of the assertion is given by Corollary 4.6, For the "only if" part, let $n$ be the order of some element of $\operatorname{Aut}(S)$. Then every prime divisor of $n$ either equals 3 , or is congruent to 1 modulo 3 by Lemma 5.2, and $n$ is not divisible by 9 by Lemma 5.3 .

\section{Subgroups}

In this section we classify finite subgroups of automorphism groups of non-trivial SeveriBrauer surfaces and prove Theorem 1.3.

Lemma 6.1. Let $\overline{\mathbb{K}}$ be an algebraically closed field of characteristic zero, and let $\check{G} \cong \boldsymbol{\mu}_{p}^{2}$, where $p$ is a prime number, be a finite subgroup of $\mathrm{GL}_{3}(\overline{\mathbb{K}})$. Suppose that $\breve{G}$ does not contain non-trivial scalar matrices. Then there exists an element of $\breve{G}$ whose fixed point locus on $\mathbb{P}_{\mathbb{\mathbb { K }}}^{2}$ contains a unique isolated point.

Proof. Since $\check{G}$ is a finite abelian group, we may assume that it consists of diagonal matrices. Let $\xi$ be a non-trivial $p$-th root of unity, and let $\hat{G}$ be the group generated by $\check{G}$ and the scalar matrix with diagonal entries equal to $\xi$. Obviously, one has $|\hat{G}|=p^{3}$, and the image of $\hat{G}$ in $\mathrm{PGL}_{3}(\overline{\mathbb{K}})$ coincides with that of $\check{G}$. Since the total number of diagonal elements of order $p$ in $\mathrm{GL}_{3}(\overline{\mathbb{K}})$ equals $p^{3}-1$, all of them must be contained in $\hat{G}$. In particular, this applies to the matrix

$$
A=\left(\begin{array}{lll}
\xi & 0 & 0 \\
0 & 1 & 0 \\
0 & 0 & 1
\end{array}\right)
$$

The fixed locus of the image of $A$ in $\mathrm{PGL}_{3}(\overline{\mathbb{K}})$ is a union of a line and an isolated fixed point in $\mathbb{P}_{\mathbb{K}}^{2}$.

Corollary 6.2. Let $p \neq 3$ be a prime number, and let $S$ be a Severi-Brauer surface over a field $\mathbb{K}$ of characteristic zero such that the group $\operatorname{Aut}(S)$ contains a subgroup $G \cong \boldsymbol{\mu}_{p}^{2}$. Then $S \cong \mathbb{P}^{2}$. In other words, for $p \geqslant 3$ every finite abelian -group acting on a non-trivial Severi-Brauer surface over a field of characteristic zero is cyclic.

Proof. Consider the action of $G$ on $S_{\overline{\mathbb{K}}} \cong \mathbb{P}_{\overline{\mathbb{K}}}^{2}$. Consider the natural projection

$$
\pi: \mathrm{SL}_{3}(\overline{\mathbb{K}}) \rightarrow \operatorname{Aut}\left(\mathbb{P}_{\overline{\mathbb{K}}}^{2}\right) \cong \mathrm{PGL}_{3}(\overline{\mathbb{K}}) \text {. }
$$

Set $\tilde{G}=\pi^{-1}(G)$, so that $\tilde{G}$ is a group of order $3 p^{2}$. Let $\check{G}$ be the $p$-Sylow subgroup of $\tilde{G}$. Then $\pi$ gives an isomorphism between $\check{G}$ and $G$. Moreover, since $p \neq 3$, the group $\check{G}$ does not contain non-trivial scalar matrices. By Lemma 6.1 there exists an element $g \in G$ such 
that the set of fixed points of $g$ on $\mathbb{P}_{\mathbb{K}}^{2}$ contains a unique isolated point. Thus the required assertion follows from Lemma 5.1.

Corollary 6.3. Let $S$ be a Severi-Brauer surface over a field $\mathbb{K}$ of characteristic zero such that the group $\operatorname{Aut}(S)$ contains a subgroup $G \cong \boldsymbol{\mu}_{3}^{2}$. Suppose that $G$ has at least one fixed point on $S_{\overline{\mathbb{K}}} \cong \mathbb{P}_{\overline{\mathbb{K}}}^{2}$. Then $S \cong \mathbb{P}^{2}$.

Proof. Consider the natural projection

$$
\pi: \mathrm{SL}_{3}(\overline{\mathbb{K}}) \rightarrow \mathrm{PGL}_{3}(\overline{\mathbb{K}})
$$

Set $\tilde{G}=\pi^{-1}(G)$, so that $\tilde{G}$ is a group of order 27 . Since $G$ has a fixed point on $\mathbb{P}_{\overline{\mathbb{K}}}^{2}$, the natural three-dimensional representation $V$ of $\tilde{G}$ is reducible. Recall that the dimension of every irreducible representation of $\tilde{G}$ either equals 1 , or is divisible by 3 . Hence $V$ splits into a sum of three one-dimensional representations of $\tilde{G}$, which means that the group $\tilde{G}$ is abelian. Choose the elements $\tilde{g}_{1}$ and $\tilde{g}_{2}$ in $\tilde{G}$ whose images in $\mathrm{PGL}_{3}(\overline{\mathbb{K}})$ generate the group $G$. Then $\tilde{g}_{1}$ and $\tilde{g}_{2}$ generate a subgroup $\check{G} \cong \boldsymbol{\mu}_{3}^{2}$ in $\tilde{G}$ such that $\check{G}$ does not contain non-trivial scalar matrices. By Lemma 6.1 there exists an element $g \in G$ such that the set of fixed points of $g$ on $\mathbb{P}_{\mathbb{K}}^{2}$ contains a unique isolated point. Thus the required assertion follows from Lemma 5.1 .

Corollary 6.4 (cf. [Sh20a, Lemma 4.1(iii),(iv), Lemma 4.6]). Let $S$ be a Severi-Brauer surface over a field $\mathbb{K}$ of characteristic zero such that the group $\operatorname{Aut}(S)$ contains a finite 3 -group $G$. Suppose that $G$ is not a subgroup of $\boldsymbol{\mu}_{3}^{2}$. Then $S \cong \mathbb{P}^{2}$.

Proof. Suppose that the Severi-Brauer surface $S$ is non-trivial. Since $G$ is a 3-group of order at least 27 , it contains a subgroup $G^{\prime}$ of order exactly 27 . Furthermore, the group $G$, and thus also $G^{\prime}$, does not contain elements of order greater than 3 by Lemma 5.3 , Therefore, if $G^{\prime}$ is abelian, then $G^{\prime} \cong \boldsymbol{\mu}_{3}^{3}$; if $G^{\prime}$ is non-abelian, then it is the Heisenberg group of order 27 and exponent 3 . In both cases $G^{\prime}$ contains a subgroup $Z \cong \boldsymbol{\mu}_{3}$ that commutes with every element of $G^{\prime}$.

We claim that $G^{\prime}$ contains a subgroup $\boldsymbol{\mu}_{3}^{2}$ acting on $S_{\overline{\mathbb{K}}} \cong \mathbb{P}_{\overline{\mathbb{K}}}^{2}$ with a fixed point. Indeed, let $g_{1}$ be a generator of $Z$, let $g_{2}$ be an element of $G^{\prime}$ not contained in $Z$, and let $g_{3}$ be an element of $G^{\prime}$ not contained in the subgroup $\boldsymbol{\mu}_{3}^{2}$ generated by $g_{1}$ and $g_{2}$. According to Lemma 5.1, the element $g_{1}$ has exactly three fixed points $P_{1}, P_{2}$, and $P_{3}$ on $\mathbb{P}_{\mathbb{K}}^{2}$. Since $g_{2}$ commutes with $g_{1}$, it preserves the set $\left\{P_{1}, P_{2}, P_{3}\right\}$. Therefore, $g_{2}$ either fixes each of the points $P_{i}$, or permutes them transitively. In the former case the group $\boldsymbol{\mu}_{3}^{2}$ generated by $g_{1}$ and $g_{2}$ acts on $\mathbb{P}_{\mathbb{K}}^{2}$ with fixed points. So, we may assume that $g_{2}$ defines a cyclic permutation of the points $P_{1}, P_{2}$, and $P_{3}$. Similarly, the element $g_{3}$ preserves the set $\left\{P_{1}, P_{2}, P_{3}\right\}$, and we may assume that it defines a cyclic permutation of $P_{1}, P_{2}$, and $P_{3}$. This means that either the element $g_{2} g_{3}$ or the element $g_{2} g_{3}^{-1}$ preserves the points $P_{1}, P_{2}$, and $P_{3}$. Together with $g_{1}$ this element generates a group $\boldsymbol{\mu}_{3}^{2}$ acting on $\mathbb{P}_{\overline{\mathbb{K}}}^{2}$ with a fixed point.

Now Corollary 6.3 shows that $S \cong \mathbb{P}^{2}$.

While the group $\boldsymbol{\mu}_{3}^{2}$ can act on a non-trivial Severi-Brauer surface over a field of characteristic zero, it appears that such an action imposes strong restrictions on other finite groups acting on this Severi-Brauer surface. 
Lemma 6.5. Let $p>3$ be a prime number, and let $S$ be a Severi-Brauer surface over a field $\mathbb{K}$ of characteristic zero such that the group $\operatorname{Aut}(S)$ contains a subgroup $G \cong \boldsymbol{\mu}_{3}^{2} \times \boldsymbol{\mu}_{p}$. Then $S \cong \mathbb{P}^{2}$.

Proof. Suppose that the Severi-Brauer surface $S$ is non-trivial. The group $\boldsymbol{\mu}_{3}^{2} \subset G$ contains four subgroups isomorphic to $\boldsymbol{\mu}_{3}$. According to Lemma [5.1, each of these subgroups has exactly three fixed points on $S_{\overline{\mathbb{K}}}$. Furthermore, none of the points fixed by two different subgroups $\boldsymbol{\mu}_{3}$ can coincide with each other, since otherwise $S \cong \mathbb{P}^{2}$ by Corollary 6.3, On the other hand, since the group $\boldsymbol{\mu}_{p} \subset G$ commutes with $\boldsymbol{\mu}_{3}^{2}$, it maps the fixed points of every element $g$ of $\boldsymbol{\mu}_{3}^{2}$ again to points fixed by $g$. Therefore, $\boldsymbol{\mu}_{p}$ has four distinct invariant sets of three points on $S_{\overline{\mathbb{K}}} \cong \mathbb{P}_{\overline{\mathbb{K}}}^{2}$. Since $p>3$, each of the 12 points in the union of these sets is $\boldsymbol{\mu}_{p}$-invariant. This is impossible by Lemma 5.1.

The next lemma shows that non-trivial non-balanced semidirect products do not appear as subgroups of automorphism groups of Severi-Brauer surfaces over fields of characteristic zero.

Lemma 6.6 (cf. Remark 4.4). Let $n$ be a positive integer divisible only by primes congruent to 1 modulo 3 , and let $G$ be a semidirect product of $\boldsymbol{\mu}_{n}$ and $\boldsymbol{\mu}_{3}$. Let $\overline{\mathbb{K}}$ be an algebraically closed field of characteristic zero. Suppose that $G \subset \mathrm{PGL}_{3}(\overline{\mathbb{K}})$. Then $G$ is either isomorphic to $\boldsymbol{\mu}_{n} \times \boldsymbol{\mu}_{3}$, or is a balanced semidirect product.

Proof. Assume that $G$ is a non-balanced semidirect product. By Lemma 2.6, for some positive integers $n_{1}$ and $n_{2}$ such that $n_{1} n_{2}=n$ and $n_{2}>1$ there is an isomorphism

$$
G \cong G_{1} \times \boldsymbol{\mu}_{n_{2}},
$$

where $G_{1} \cong \boldsymbol{\mu}_{n_{1}} \rtimes \boldsymbol{\mu}_{3}$. Let us also assume that $G$ is not isomorphic to $\boldsymbol{\mu}_{n} \times \boldsymbol{\mu}_{3}$. Then $G$, and thus also $G_{1}$, is not abelian; in particular, we have $n_{1}>1$.

Consider the projection

$$
\pi: \mathrm{SL}_{3}(\overline{\mathbb{K}}) \rightarrow \mathrm{PGL}_{3}(\overline{\mathbb{K}}),
$$

and set $\tilde{G}_{1}=\pi^{-1}\left(G_{1}\right)$. The group $\tilde{G}_{1}$ is not abelian, and its order $3 n_{1}$ is not divisible by 2 . Thus the natural three-dimensional representation of $\tilde{G}_{1}$ is irreducible.

Let $g$ be a generator of the subgroup $\boldsymbol{\mu}_{n_{2}} \subset G$, and choose a preimage $\tilde{g}$ of $g$ with respect to $\pi$. Since the order $n_{2}$ of $g$ is coprime to 3 , we can multiply $\tilde{g}$ by an appropriate scalar matrix and assume that the order of $\tilde{g}$ equals $n_{2}$ as well. We claim that $\tilde{g}$ commutes with $\tilde{G}_{1}$. Indeed, let $\gamma$ be an element of $\tilde{G}_{1}$. Since $\pi(\gamma) \in G_{1}$ commutes with $\pi(\tilde{g})=g$, the commutator $\kappa=\tilde{g} \gamma \tilde{g}^{-1} \gamma^{-1}$ is a scalar matrix in $\mathrm{SL}_{3}(\overline{\mathbb{K}})$. On the other hand, $\tilde{g}^{n_{2}}$ is the identity matrix, so it commutes with $\gamma$. Writing

$$
\tilde{g}^{n_{2}} \gamma=\kappa^{n_{2}} \gamma \tilde{g}^{n_{2}},
$$

one concludes that $\kappa^{n_{2}}$ is the identity matrix, and thus $\kappa$ is the identity matrix itself.

We see that $\tilde{g}$ commutes with $\tilde{G}_{1}$. Therefore, by Schur's lemma $\tilde{g}$ acts in the threedimensional representation by a scalar matrix. This is impossible, because the image $g$ of $\tilde{g}$ in $\mathrm{PGL}_{3}(\overline{\mathbb{K}})$ is non-trivial. The obtained contradiction shows that $G \cong \boldsymbol{\mu}_{n} \times \boldsymbol{\mu}_{3}$.

Remark 6.7. For an alternative proof of Lemma 6.6. consider two elements $g_{1}, g_{2} \in \mathrm{PGL}_{3}(\overline{\mathbb{K}})$ such that the orders of $g_{i}$ are finite, at least one of the orders is greater than 3, and the fixed point locus Fix $\left(g_{i}\right)$ of each $g_{i}$ consists of three distinct points. One can notice that $g_{1}$ and $g_{2}$ commute with each other if and only 
if $\operatorname{Fix}\left(g_{1}\right)=\operatorname{Fix}\left(g_{2}\right)$. Applying this criterion to the generators $g_{2}$ and $g$ of the subgroups $\boldsymbol{\mu}_{n_{2}}$ and $\boldsymbol{\mu}_{3}$ of $G$ appearing in (2.1) (and assuming that $\operatorname{Fix}\left(g_{2}\right)$ and $\operatorname{Fix}(g)$ consist of three points), and then to $g$ and a generator of the subgroup $\boldsymbol{\mu}_{n}$ of $G$, we conclude that $G$ is isomorphic to a direct product of $\boldsymbol{\mu}_{n}$ and $\boldsymbol{\mu}_{3}$. The case when the fixed locus of some of the elements $g_{2}$ or $g$ consists of a line and an isolated point is also easy to analyze.

Let us summarize the above results.

Corollary 6.8. There exists a field $\mathbb{K}$ of characteristic zero and a non-trivial SeveriBrauer surface over $\mathbb{K}$ such that the group $\operatorname{Aut}(S)$ contains a finite subgroup isomorphic to $G$ if and only if there is a positive integer $n$ divisible only by primes congruent to 1 modulo 3 such that $G$ is isomorphic either to $\boldsymbol{\mu}_{n}$, or to $\boldsymbol{\mu}_{3 n}$, or to a balanced semidirect product $\boldsymbol{\mu}_{n} \rtimes \boldsymbol{\mu}_{3}$, or to the direct product $\boldsymbol{\mu}_{3} \times\left(\boldsymbol{\mu}_{n} \rtimes \boldsymbol{\mu}_{3}\right)$, where the semidirect product is balanced.

Proof. The "if" part of the assertion is given by Corollary 4.6 and Lemmas 4.3 and 4.5 . Let us prove the "only if" part. Let $S$ be a non-trivial Severi-Brauer surface over a field of characteristic zero, and let $G$ be a finite subgroup of $\operatorname{Aut}(S)$.

To start with, assume that $G$ is abelian. Then $G$ is isomorphic to the product of its $p$-Sylow subgroups $G(p)$ for all $p$ dividing $|G|$. By Corollary 6.2, for $p \neq 3$ the group $G(p)$ is cyclic. By Corollary 6.4, the group $G(3)$ is isomorphic to a subgroup of $\boldsymbol{\mu}_{3}^{2}$. Moreover, if $G(3) \cong \boldsymbol{\mu}_{3}^{2}$, then all the groups $G(p)$ for $p \neq 3$ are trivial by Lemma 6.5. We conclude that $G$ is isomorphic either to $\boldsymbol{\mu}_{3}^{2}$, or to $\boldsymbol{\mu}_{n}$, or to $\boldsymbol{\mu}_{3 n}$, where $n \geqslant 1$ is the product of $|G(p)|$ for all prime divisors of $|G|$ different from 3 . In the former case, we can consider $G \cong \boldsymbol{\mu}_{3}^{2}$ as a product of $\boldsymbol{\mu}_{3}$ and a balanced semidirect product $\boldsymbol{\mu}_{1} \rtimes \boldsymbol{\mu}_{3} \cong \boldsymbol{\mu}_{3}$. In the latter two cases, all prime divisors $p$ of $n$ are congruent to 1 modulo 3 by Lemma 5.2. This proves the required assertion in the case when $G$ is abelian.

Now assume that $G$ is not abelian. By Theorem 1.1, the group $G$ contains a normal abelian subgroup $H$ of index 3. Since $G$ does not contain elements of order 9 by Lemma 5.3, one has $G \cong H \rtimes \boldsymbol{\mu}_{3}$ by Lemma 2.9. According to the above argument, $H$ is isomorphic either to $\boldsymbol{\mu}_{3}^{2}$, or to $\boldsymbol{\mu}_{n}$, or to $\boldsymbol{\mu}_{3 n}$, where $n$ is divisible only by primes congruent to 1 modulo 3. In the first case $|G|=3|H|=27$, which is impossible by Corollary 6.4. In the second case we have $G \cong \boldsymbol{\mu}_{n} \rtimes \boldsymbol{\mu}_{3}$, and $G$ is not isomorphic to $\boldsymbol{\mu}_{n} \times \boldsymbol{\mu}_{3}$ because it is non-abelian. Thus $G$ is a balanced semidirect product by Lemma 6.6. In the third case

$$
G \cong \boldsymbol{\mu}_{3 n} \rtimes \boldsymbol{\mu}_{3} \cong\left(\boldsymbol{\mu}_{3} \times \boldsymbol{\mu}_{n}\right) \rtimes \boldsymbol{\mu}_{3} \cong \boldsymbol{\mu}_{3} \times\left(\boldsymbol{\mu}_{n} \rtimes \boldsymbol{\mu}_{3}\right),
$$

because there are no non-trivial homomorphisms from $\boldsymbol{\mu}_{3}$ to $\boldsymbol{\mu}_{3}^{*} \cong \boldsymbol{\mu}_{2}$. Furthermore, the semidirect product $\boldsymbol{\mu}_{n} \rtimes \boldsymbol{\mu}_{3}$ on the right hand side of (6.1) is not a direct product, because the group $G$ is non-abelian. Hence this semidirect product is balanced by Lemma 6.6. This completes the proof in the case when $G$ is non-abelian.

The following assertion is implied by the results of [Sh20a] and [Sh20c].

Proposition 6.9. Let $S$ be a non-trivial Severi-Brauer surface over a field of characteristic zero, and let $G$ be a finite subgroup of $\operatorname{Bir}(S)$. Then either $G$ is isomorphic to a subgroup of $\operatorname{Aut}(S)$, or $G$ is a subgroup of $\boldsymbol{\mu}_{3}^{3}$. Furthermore, there exists a non-trivial Severi-Brauer surface over a field of characteristic zero whose birational automorphism group contains a subgroup isomorphic to $\boldsymbol{\mu}_{3}^{3}$. 
Proof. By [Sh20a, Proposition 3.7], the group $G$ is either a subgroup of $\boldsymbol{\mu}_{3}^{3}$, or is isomorphic to a subgroup of $\operatorname{Aut}(S)$, or is isomorphic to a subgroup of $\operatorname{Aut}\left(S^{o p}\right)$, where $S^{o p}$ is the Severi-Brauer surface corresponding to the central simple algebra opposite to the one corresponding to $S$. In the latter case $G$ is also isomorphic to a subgroup of $\operatorname{Aut}(S)$ by [Sh20a, Corollary 4.5]. A Severi-Brauer surface $S$ with $\boldsymbol{\mu}_{3}^{3} \subset \operatorname{Bir}(S)$ exists by [Sh20c, Theorem 1.2].

Now we are ready to prove our main result.

Proof of Theorem 1.3. Assertion (i) is given by Corollary 5.4 and Proposition 6.9, Assertion (ii) is given by Corollary 6.8. Assertion (iii) follows from Proposition 6.9.

Remark 6.10. There is a number of results on finite subgroups of multiplicative groups of division algebras, starting from the classical papers [Her53] and Ami55]. They can hardly be used to conclude anything about automorphism groups of Severi-Brauer varieties, since a finite group in the quotient $A^{*} / \mathbb{K}^{*}$ is not necessarily an image of a finite subgroup of $A^{*}$. However, every finite subgroup in $A^{*}$ projects to a finite subgroup of $A^{*} / \mathbb{K}^{*}$. It would be interesting to find out which results on finite subgroups of multiplicative groups of division algebras can be recovered from geometric properties of Severi-Brauer varieties.

\section{Field OF RATiOnAL NUMBERS}

In this section we discuss finite groups acting by birational automorphisms of non-trivial Severi-Brauer surfaces over $\mathbb{Q}$ and prove Corollary 1.4.

It is well known that the group $\mathrm{PGL}_{3}(\mathbb{Q})$ does not contain elements of prime order $p \geqslant 5$, see e.g. [DI09b, §1]. The following assertion is a generalization of this result for automorphism groups of Severi-Brauer surfaces.

Lemma 7.1. Let $S$ be a Severi-Brauer surface over the field $\mathbb{Q}$. Then the group Aut $(S)$ does not contains elements of prime order $p \geqslant 5$.

We give two slightly different proofs of Lemma 7.1. The first of them uses the same approach as the proofs of Lemmas 5.2 and 5.3 .

First proof of Lemma 7.1. Suppose that there is an element $g \in \operatorname{Aut}(S)$ of order $p \geqslant 5$. We may assume that the Severi-Brauer surface $S$ is non-trivial. Consider the action of $g$ on $S_{\overline{\mathbb{Q}}} \cong \mathbb{P}_{\overline{\mathbb{Q}}}^{2}$. Let $\tilde{g}$ be a preimage of $g$ under the natural projection

$$
\pi: \mathrm{SL}_{3}(\overline{\mathbb{Q}}) \rightarrow \mathrm{PGL}_{3}(\overline{\mathbb{Q}}) \text {. }
$$

Since $p$ is not divisible by 3 , we can multiply $\tilde{g}$ by an appropriate scalar matrix so that the order of $\tilde{g}$ equals $p$. Moreover, the element $\tilde{g}$ of order $p$ such that $\pi(\tilde{g})=g$ is unique in $\mathrm{SL}_{3}(\overline{\mathbb{Q}})$, and hence it is defined over the field $\mathbb{Q}$.

Let $\xi_{1}, \xi_{2}$, and $\xi_{3}$ be the eigen-values of $\tilde{g}$; these are $p$-th roots of unity. According to Lemma 5.1, the element $g$ has exactly three fixed points on $\mathbb{P}_{\mathbb{Q}}^{2}$, and these points are transitively permuted by the group $\operatorname{Gal}(\overline{\mathbb{Q}} / \mathbb{Q})$. Thus the roots of unity $\xi_{1}, \xi_{2}$, and $\xi_{3}$ are pairwise different and form a $\operatorname{Gal}(\overline{\mathbb{Q}} / \mathbb{Q})$-orbit. However, the group Gal $(\overline{\mathbb{Q}} / \mathbb{Q})$ acts transitively on the $p-1$ primitive roots of unity of degree $p$ by Theorem 3.5. Since $p-1>3$, this gives a contradiction.

The second proof uses the properties of central simple algebras. 
Second proof of Lemma 7.1. Suppose that there is an element $g \in \operatorname{Aut}(S)$ of order $p \geqslant 5$. We may assume that the Severi-Brauer surface $S$ is non-trivial. Let $A$ be the central simple algebra of dimension 9 over $\mathbb{Q}$ corresponding to the surface $S$. By Lemma 3.1 there exists an element $x \in A^{*}$ such that $x \notin \mathbb{Q}$ and $x^{p}=b$ for some $b \in \mathbb{Q}$.

Since $S$ is a non-trivial Severi-Brauer surface, $A$ is a division algebra by Lemma 3.4 . Thus $x$ generates a subfield $\mathbb{L} \supsetneq \mathbb{Q}$ inside $A$. The field $\mathbb{L}$ is contained in some maximal subfield of $A$. On the other hand, any maximal subfield of $A$ has degree 3 over $\mathbb{Q}$, see [Bou58, §VIII.10.3]. Hence $\mathbb{L}$ also has degree 3 over $\mathbb{Q}$ (and is maximal itself). Therefore, there exists an irreducible polynomial $F(t)$ of degree 3 over $\mathbb{Q}$ such that $x$ is a root of $F(t)$. Thus $F(t)$ divides the polynomial $t^{p}-b$. Note that if $b$ is not a $p$-th power of a rational number, then the polynomial $t^{p}-b$ is irreducible over $\mathbb{Q}$, see for instance [Lan65, Theorem VIII.9.16]. Since $p>3$ by assumption, one cannot have $F(t)=t^{p}-b$, and hence the polynomial $t^{p}-b$ must be reducible. Thus we see that

$$
b=c^{p}
$$

for some $c \in \mathbb{Q}$, and the roots of $F(t)$ have the form $x=\xi_{1} c, \xi_{2} c$, and $\xi_{3} c$, where $\xi_{1}, \xi_{2}$, and $\xi_{3}$ are pairwise different $p$-th roots of unity. We see that $\xi_{1}, \xi_{2}$, and $\xi_{3}$ form a $\operatorname{Gal}(\overline{\mathbb{Q}} / \mathbb{Q})$-orbit. This is impossible by Theorem 3.5. Alternatively, one can conclude from (17.1) that the polynomial $F(c t)$ divides the $p$-th cyclotomic polynomial $\Phi_{p}(t)$, and use the fact that the latter is irreducible over $\mathbb{Q}$.

For an alternative proof (and a more general statement) of Lemma 7.1 we refer the reader to [Ser07, Theorem 6].

Finally, we prove Corollary 1.4

Proof of Corollary 1.4. By Proposition 6.9, we may assume that the group $G$ is contained in $\operatorname{Aut}(S)$. Then the order of $G$ is divisible only by primes not exceeding 3 by Lemma 7.1 , On the other hand, we know from Theorem 1.3(i) (or from Lemma 5.2) that the order of $G$ is odd. Therefore, in this case $G$ is a 3-group, and thus it is a subgroup of $\boldsymbol{\mu}_{3}^{2}$ by Corollary 6.4.

Remark 7.2. Using the same arguments as in the proofs of Lemma 7.1 and Corollary 1.4, one can generalize both of these assertions to arbitrary fields that contain no roots of unity of any prime degree $p \geqslant 5$.

\section{REFERENCES}

[Ami55] S. Amitsur. Finite subgroups of division rings. Trans. Amer. Math. Soc. 80 (1955), 361-386.

[Art82] M. Artin. Brauer-Severi varieties. In Brauer groups in ring theory and algebraic geometry (Wilrijk, 1981), volume 917 of Lecture Notes in Math., pp. 194-210. Springer, Berlin-New York, 1982.

[Bea10] A. Beauville. Finite subgroups of $\mathrm{PGL}_{2}(K)$. In Vector bundles and complex geometry, pp. 23-29. Contemp. Math. 522, Amer. Math. Soc., Providence, RI, 2010.

[BE99] H. Besche, B. Eick. Construction of finite groups. J. Symbolic Comput. 27 (1999), no. 4, $387-404$.

[Bou58] N. Bourbaki. Éléments de mathématique. 23. Première partie: Les structures fondamentales de l'analyse. Livre II: Algèbre. Chapitre 8: Modules et anneaux semi-simples. Actualités Sci. Ind., no. 1261, Hermann, Paris, 1958.

[Châ44] F. Châtelet. Variations sur un thème de H. Poincaré. Ann. Sci. École Norm. Sup. (3), 61 (1944), 249-300. 
[CF67] Algebraic number theory. Edited by J. W. S. Cassels and A. Fröhlich. Academic Press, London; Thompson Book Co., Inc., Washington, D.C., 1967.

[DI09a] I. Dolgachev, V. Iskovskikh. Finite subgroups of the plane Cremona group. In Algebra, arithmetic, and geometry: in honor of Yu. I. Manin. Vol. I, pp. 443-548, Progr. Math. 269, Birkhäuser Boston, Boston, MA, 2009.

[DI09b] I. Dolgachev, V. Iskovskikh. On elements of prime order in the plane Cremona group over a perfect field. Int. Math. Res. Not. IMRN 2009, no. 18, 3467-3485.

[GA13] M. Garcia-Armas. Finite group actions on curves of genus zero. J. Algebra, 394 (2013), 173-181.

[GS06] Ph. Gille, T. Szamuely. Central simple algebras and Galois cohomology. Cambridge Studies in Advanced Mathematics, 101. Cambridge University Press, Cambridge, 2006.

[GSh18] S. Gorchinskiy, C. Shramov. Unramified Brauer group and its applications. Translations of Mathematical Monographs, 246. American Mathematical Society, Providence, RI, 2018.

[Her53] I. Herstein. Finite multiplicative subgroups in division rings. Pacific J. Math. 3 (1953), 121126.

[Isa08] M. Isaacs. Finite group theory. Graduate Studies in Mathematics, 92. AMS, Providence, RI, 2008.

[Kol16] J. Kollár. Severi-Brauer varieties; a geometric treatment. arXiv:1606.04368 (2016)

[Lan65] S. Lang. Algebra. Addison-Wesley Publishing Co., Inc., Reading, Mass., 1965.

[Pop14] V. Popov. Jordan groups and automorphism groups of algebraic varieties. in Automorphisms in birational and affine geometry, pp. 185-213, Springer Proc. Math. Stat., 79, Springer, Cham, 2014.

[Ser07] J.-P. Serre. Bounds for the orders of the finite subgroups of $G(k)$. Group representation theory, 405-450, EPFL Press, Lausanne, 2007.

[Sh20a] C. Shramov. Birational automorphisms of Severi-Brauer surfaces. Sb. Math., 211 (2020), no. $3,466-480$.

[Sh20b] C. Shramov. Non-abelian groups acting on Severi-Brauer surfaces. arXiv:2006.01214 (2020)

[Sh20c] C. Shramov. Automorphisms of cubic surfaces without points. arXiv:2006.02531 (2020)

[ShV20] C. Shramov, V. Vologodsky. Boundedness for finite subgroups of linear algebraic groups. arXiv:2009.14485 (2020)

[Ste89] L. Stern. On the norm groups of global fields. J. Number Theory 32 (1989), no. 2, 203-219.

[Wed21] J. Wedderburn. On division algebras. Trans. Amer. Math. Soc. 22 (1921), no. 2, 129-135.

[Yas17] E. Yasinsky. The Jordan constant for Cremona group of rank 2. Bull. Korean Math. Soc. 54 (2017), no. 5, 1859-1871.

Steklov Mathematical Institute of Russian Academy of Sciences, 8 Gubkina st., Moscow, 119991, Russia

National Research University Higher School of Economics, Laboratory of Algebraic

Geometry, 6 Usacheva str., Moscow, 119048, Russia

Email address: costya.shramov@gmail.com 